\title{
Criminologie
}

\section{Homme à femmes : le proxénète et sa place parmi les prostituées}

\section{Isa Savoie-Gargiso et Carlo Morselli}

Volume 46, numéro 1, printemps 2013

URI : https://id.erudit.org/iderudit/1015301ar

DOI : https://doi.org/10.7202/1015301ar

Aller au sommaire du numéro

\section{Éditeur(s)}

Les Presses de l’Université de Montréal

ISSN

0316-0041 (imprimé)

1492-1367 (numérique)

Découvrir la revue

Citer cet article

Savoie-Gargiso, I. \& Morselli, C. (2013). Homme à femmes : le proxénète et sa place parmi les prostituées. Criminologie, 46(1), 243-268.

https://doi.org/10.7202/1015301ar
Résumé de l'article

La présente étude se penche sur les rôles, les interactions et l'échange des ressources au sein d'un réseau de prostitution démantelé par un service de police d'une grande ville canadienne. Plutôt que d'inscrire le débat dans la perspective de l'exploitation des prostituées par les proxénètes, l'analyse de ce réseau est réalisée dans une perspective du partage des ressources, qui sont plus souvent utilisées pour d'autres types de réseaux criminels. Cette perspective tient compte de la façon dont une prostituée est dominée ou pas par son proxénète et de quelle manière cela détermine le rôle de celui-ci dans le processus d'échange. Le message implicite est que les prostituées peuvent avoir un certain contrôle sur la manière dont les autres les contrôlent. Une analyse des positions des individus du réseau est appliquée afin de cibler les participants les plus centraux et stratégiques. Une analyse des conversations est mise de l'avant comme méthode plus poussée d'analyse des réseaux criminels, ce qui permet d'apprécier le rôle de chaque prostituée et de chaque proxénète ainsi que leur façon d'interagir. L'étude conclut que les proxénètes, entre eux, sont plutôt axés sur l'entraide alors que les prostituées sont en constante compétition. Finalement, même si les proxénètes utilisent le capital des prostituées afin de forger le leur, ces dernières sont d'autant plus indispensables qu'un proxénète sans prostituées ne peut exister alors que l'inverse est possible. Cette réalité procure ainsi aux prostituées une forme de pouvoir non négligeable dans leurs relations avec leur proxénète.
Ce document est protégé par la loi sur le droit d'auteur. L’utilisation des services d’Érudit (y compris la reproduction) est assujettie à sa politique d'utilisation que vous pouvez consulter en ligne.

https://apropos.erudit.org/fr/usagers/politique-dutilisation/ 


\title{
Homme à femmes: le proxénète et sa place parmi les prostituées
}

\author{
Is a Savoie-Gargiso \\ Diplômée à la maîtrise en criminologie \\ École de criminologie, Université de Montréal \\ isa.savoie-gargiso@rcmp-grc.gc.ca \\ Carlo Morselli \\ Professeur, École de criminologie/CICC, Université de Montréal \\ carlo.morselli@umontreal.
}

\begin{abstract}
RÉSUMÉ - La présente étude se penche sur les rôles, les interactions et l'échange des ressources au sein d'un réseau de prostitution démantelé par un service de police d'une grande ville canadienne. Plutôt que d'inscrire le débat dans la perspective de l'exploitation des prostituées par les proxénètes, l'analyse de ce réseau est réalisée dans une perspective du partage des ressources, qui sont plus souvent utilisées pour d'autres types de réseaux criminels. Cette perspective tient compte de la façon dont une prostituée est dominée ou pas par son proxénète et de quelle manière cela détermine le rôle de celui-ci dans le processus d'échange. Le message implicite est que les prostituées peuvent avoir un certain contrôle sur la manière dont les autres les contrôlent. Une analyse des positions des individus du réseau est appliquée afin de cibler les participants les plus centraux et stratégiques. Une analyse des conversations est mise de l'avant comme méthode plus poussée d'analyse des réseaux criminels, ce qui permet d'apprécier le rôle de chaque prostituée et de chaque proxénète ainsi que leur façon d'interagir. L'étude conclut que les proxénètes, entre eux, sont plutôt axés sur l'entraide alors que les prostituées sont en constante compétition. Finalement, même si les proxénètes utilisent le capital des prostituées afin de forger le leur, ces dernières sont d'autant plus indispensables qu'un proxénète sans prostituées ne peut exister alors que l'inverse est possible. Cette réalité procure ainsi aux prostituées une forme de pouvoir non négligeable dans leurs relations avec leur proxénète.
\end{abstract}

MOTS-CLÉS - Proxénète, prostitution, réseau criminel, analyse de réseaux, analyse des conversations. 


\section{Introduction $^{1}$}

Les recherches sur la présence des proxénètes dans le milieu de la prostitution varient; certaines perçoivent le proxénète comme un exploiteur et d'autres le perçoivent comme un entrepreneur travaillant de concert avec les prostituées. Si division il y a quant à l'interprétation de la place des proxénètes parmi les prostituées, il y a tout de même un consensus sur la définition du proxénète, c'est-à-dire un individu contrôlant les actions et vivant des fruits du travail d'une ou plusieurs femmes se prostituant. Effectivement, c'est dans le sens attribué à la notion de contrôle qu'est la source du débat entre les différentes images du proxénète, qu'il soit considéré comme étant un protecteur ou un exploiteur.

Certains chercheurs considèrent les prostituées comme étant des victimes, consentantes ou non, d'hommes qui les exploitent. Dans ce contexte, la prostitution n'est pas considérée comme un travail, mais plutôt comme une façon, pour les hommes, d'exercer un pouvoir qui se traduit par une exploitation sexuelle et souvent par la violence qui est une réalité indéniable dans ce genre de relations (Barry, 1995; Hodgson, 1997; Norton-Hawk, 2004). Cette perspective a maintes fois été dénoncée comme étant moralisatrice et comme ne permettant pas aux femmes s'adonnant à la prostitution de se responsabiliser puisqu'elles sont perçues comme d'éternelles victimes de la domination masculine (Bell, 1987).

Des organisations de défense des droits des travailleuses du sexe ont vu le jour afin de contester cette victimisation des prostituées. Les services sexuels offerts sont le produit d'un emploi, au même titre que n'importe quel autre emploi (Svedgi et al., 2002). Pour celles-ci, ce n'est pas dans l'offre de services sexuels que se trouve la source du problème, mais plutôt dans les conditions de travail reliées à la prostitution, par exemple la violence des clients, les contraventions et autres peines possibles. En effet, par sa nature illégale et peu reconnue, le travail du sexe se réalise souvent dans des conditions plutôt précaires et dangereuses (Bell, 1987; Lacasse, 2003). La décriminalisation du travail du sexe pourrait réduire la fréquence des situations à risque ainsi que le stigmate associé aux femmes pratiquant ce métier. Globalement, ce courant ne met pas l'accent sur la victimisation de la prostituée par l'homme, mais bien

1. Nous tenons à remercier Pierre Tremblay, Martin Bouchard et Chloé Leclerc pour leurs commentaires à diverses étapes de la rédaction de cet article. 
sur la prise de pouvoir (empowerment) de celle-ci sur ses conditions de vie.

Que l'on perçoive la prostitution ou le travail du sexe comme étant une pratique légitime ou pas, qu'une prostituée ou une travailleuse du sexe soit perçue comme une victime ou comme une travailleuse, l'exploitation de celle-ci peut être directement liée aux pratiques du proxénétisme. Cette exploitation se traduit par des pressions ou de la violence visant à obliger une prostituée à travailler afin d'obtenir toujours plus de gains ou par la façon dont certaines sont manipulées (Hodgson, 1997; Williamson et Cluse-Tolar, 2002; Dalla, 2003).

Plusieurs études ont témoigné de la violence que subissent les prostituées de la part des clients ainsi que de leur proxénète. L'étude de Raphael et Shapiro (2004) indique que la moitié des prostituées rencontrées $(n=222)$, qu'elles travaillent dans le milieu de la prostitution à l'intérieur (salon de massage, service d'escorte) ou bien à l'extérieur (rue), étaient victimes de violence de la part de leur proxénète et que $35 \%$ avaient été violées par ce dernier. Dans le même sens, Hodgson (1997) trouve que $85 \%$ de ses répondantes ont été violentées par leur proxénète.

Selon l'étude de May et al. (2000), les proxénètes suivent parfois un modèle bien précis de recrutement qui leur permet par la suite d'exercer leur pouvoir et leur contrôle sur leurs prostituées. Au départ, ils ciblent des adolescentes vulnérables et isolées socialement, qui proviennent souvent de familles dysfonctionnelles (McLeod, 2003; Norton-Hawk, 2004). Par la suite, ils tentent d'établir une relation de dépendance, en utilisant leur charme. Le proxénète assure alors une sécurité affective importante, qui manquait à cette jeune fille depuis un certain temps ou qu'elle n'avait jamais ressentie. Les relations qui en résultent sont souvent qualifiées d'amoureuses par les prostituées. Le fait que le proxénète est un homme qui contrôle leur vie est alors plutôt difficile à reconnaître (May et al., 2000; Williamson et Cluse-Tolar, 2002). C'est lorsqu'ils peuvent instaurer un certain contrôle qu'ils présentent subtilement l'idée des activités de prostitution, ce qui peut mener à la domination totale du proxénète sur la jeune fille.

D'autres chercheurs estiment que les prostituées peuvent retirer des gains à s'engager dans des activités de prostitution, même avec l'aide d'un proxénète (May et al., 2000; Shelby, 2002; Augustin, 2005). La relation n'est alors pas perçue comme un besoin de contrôle et de pouvoir du proxénète sur ses prostituées. Il s'agit d'une relation de travail où le 
proxénète est en quelque sorte le gérant d'une entreprise et les prostituées, ses employées. Typiquement, les proxénètes dirigent l'ensemble des décisions liées aux activités de prostitution: un quota à atteindre par jour, l'endroit où la prostituée travaillera, l'horaire de travail, le montant à demander aux clients ainsi que la façon dont l'argent amassé sera dépensé (Barry, 1995; May et al., 2000; Dalla, 2003). Le proxénète gère donc l'ensemble des conditions de travail et même des conditions de vie de la prostituée.

L'étude de Pheterson (1996) présente la décision de s'engager dans le travail du sexe comme étant rationnelle, certaines de ces femmes prenant même l'initiative d'engager un proxénète afin de combler différents besoins. Le proxénète aurait donc une fonction précise dans le milieu de la prostitution. Le besoin de protection, lié notamment au risque encouru lors des activités de prostitution, est un des plus souvent cités dans les recherches où ont été interviewées des femmes ayant un proxénète (Milner et Milner, 1972; Enablers Inc., 1978; Caplan, 1984; Weisberg, 1985; Hogdson, 1997; May et al., 2000; Svedgi et al., 2002).

\section{Le modèle du partage des ressources}

En présentant le rôle du proxénète comme étant celui d'un protecteur et non pas celui d'un exploiteur, cela ne permet pas d'expliquer pleinement sa présence, son importance dans le milieu de la prostitution. Il n'est pas non plus facile de déterminer si un proxénète est un protecteur ou un exploiteur.

Une manière de faire une intégration entre ces deux extrêmes est le modèle du partage de ressources. Ce modèle a en effet été appliqué régulièrement au trafic des stupéfiants (Adler, 1985, 1993; Haller, 1990; Jacobs, 1999; Eck et Gersh, 2000 ; Morselli, 2009 ). Ce cadre théorique positionne les participants de diverses activités criminelles dans un processus d'échange et propose que les participants joignent leurs ressources et leurs compétences afin de réaliser un but commun, car cela leur permet de maximiser leurs chances de réussite ainsi que de partager les risques de détection. La collaboration nécessaire entre les différentes personnes pour faciliter des actes criminels est la base de ce modèle.

Ces études rejettent la croyance populaire en un modèle hiérarchique ou pyramidal où les personnes dirigeantes, se trouvant bien protégées au haut de la pyramide, donnent des ordres et des directives à d'autres plus bas dans la hiérarchie, des exécutants qui sont ainsi plus vulné- 
rables. Les constats décrivent plutôt des réseaux moins structurés qui permettent des relations plus égalitaires entre des partenaires. Sans ce partage de ressources, la réalisation des activités criminelles se complexifie, devient même impossible. Cette façon d'aborder les relations à l'intérieur de réseaux est intéressante en ce qu'elle cherche à en trouver la structure et non pas à la présumer.

Les partenariats visant le partage des ressources ne sont pas limités au marché de rue ou de détail. En effet, Block (1979) a trouvé des partenariats à différents niveaux de vente de stupéfiants dans les années 1920 à New York, soit l'importation, la vente de rue, la vente au détail et les franchises. Adler $(1983,1985)$ a également observé une collaboration assez souple entre les différentes entreprises œuvrant dans le trafic de stupéfiants en Californie dans les années 1970. Pearson et Hobbs (2001), qui ont réalisé des entrevues avec des agents de justice et des prisonniers impliqués dans le trafic de stupéfiants en Grande-Bretagne, en sont venus à la conclusion qu'il est plus utile de concevoir cette activité criminelle comme étant des partenariats entre différents commerçants indépendants. Dans un contexte canadien et québécois, Desroches (2005) présente les expériences de 70 trafiquants de drogues à différents niveaux. Dans presque $30 \%$ de cet échantillon, les répondants ont indiqué avoir été impliqués dans des partenariats. Dans certains cas, cela allait même jusqu'à $50 \%$ d'implication dans un partenariat à long terme. Certains étaient moins cohésifs et représentaient plutôt des entrepreneurs indépendants qui s'alliaient pour tirer avantage des ressources de l'un et l'autre.

Le cadre dans lequel les proxénètes et les prostituées évoluent a plusieurs points communs avec le milieu des drogues illégales. Trois points clés sont à retenir pour la présente étude. Premièrement, l'illégalité des deux milieux force les participants à régler leurs conflits et à gérer leurs problèmes en dehors des structures légales qu'utilisent habituellement les personnes non déviantes. Une telle gouvernance extralégale conduit à une demande pour des protecteurs privés, une ressource que, généralement, le proxénète fournit aux prostituées. Deuxièmement, dans les deux milieux, le pouvoir et la domination sont généralement attribués au fournisseur de protection privée. Toutefois, les recherches conduites dans ces deux secteurs de la criminalité ont considérablement exagéré cette position hiérarchique. Que ce soit le proxénète, le mafioso ou le baron de la drogue, la prémisse selon laquelle ils sont des dirigeants parce qu'ils sont des protecteurs a été en grande partie réfutée face à des démonstrations consistantes et systématiques indiquant que de tels 
commerces illégaux sont généralement structurés suivant un ordre flexible et des relations informelles (Morselli, 2009). Le troisième point concerne le fort individualisme qui sous-tend la participation à ces activités illégales. Alors que l'opinion populaire voudrait nous faire croire que les trafiquants de drogue, les proxénètes et les prostituées opèrent dans des groupes fermés et loyaux, il est devenu de plus en plus évident que la participation constante dans de tels milieux est avant tout une affaire d'opportunisme. Ainsi, chaque acteur met son intérêt de l'avant dans le schéma général qui l'unit aux autres dans un réseau commun. Dans de tels contextes individualistes, ce qui peut sembler comme une relation de pouvoir en est probablement une qui s'articule autour d'un processus donnant-donnant de partage des ressources nécessaires pour poursuivre l'activité visée. Ce qu'il est facile d'oublier, et où nous pourrions apprendre des relations de clientélismes, c'est que la personne qui donne le plus et qui est perçue comme l'acteur exploité dans la relation est susceptible de développer du pouvoir au fur et à mesure que la relation se poursuit. C'est cette possible inversion des rôles qui est au cœur de la présente étude et c'est ce stéréotype qui sous-tend la relation proxénète-prostituée que nous contestons avec les analyses qui suivent. Il est possible de contrôler la façon dont les autres nous contrôlent et il n'y a pas de meilleur milieu pour observer ce processus que celui qui unit les prostituées à leur proxénète.

\section{Méthodologie}

La base empirique de cette étude s'étend à une enquête d'un réseau de proxénètes et de prostituées dans une grande ville canadienne réalisée en 2003. L'activité principale des acteurs centraux de ce réseau ciblé par l'enquête était le proxénétisme et la prostitution. Comme la majorité des prostituées de ce réseau travaillaient alors dans des bars de danseuses, le défi principal des enquêteurs du dossier était de prouver non seulement qu'elles offraient des «extras», donc de confirmer qu'elles s'adonnaient bien à la prostitution, mais également qu'elles remettaient leurs gains financiers à leur proxénète respectif. Pour se faire, l'écoute électronique fut l'ultime moyen d'enquête utilisé.

En tout, 55000 pages de transcriptions d'écoute électronique ont été amassées durant l'enquête. Les lignes téléphoniques mises sous écoute étaient celles des principaux proxénètes ciblés, de quelques prostituées, 
de quelques amis des proxénètes ainsi que celle des parents des deux principaux proxénètes.

Avec les transcriptions d'écoute électronique comme source première de données, il a été possible de construire une matrice de communication des individus ciblés lors de l'enquête. L'application de l'analyse de réseau permet de comprendre l'ensemble des interactions et les positions spécifiques des participants. Ce type d'analyse permet d'appliquer le modèle du partage des ressources retenu en cernant le processus d'échange ainsi que les individus qui ont un meilleur accès que d'autres aux ressources du réseau (Degenne et Forsé, 1994). Au départ, 202 personnes se retrouvaient dans le réseau. Toutefois, afin de réaliser cette analyse, soixante individus ont été exclus parce qu'il n'a pas été possible de les identifier malgré leur mention dans les conversations interceptées ou parce que leur importance était trop minime. Ainsi, la matrice est formée de 142 personnes. Une matrice binaire symétrique a été construite en tenant compte du contact (1) ou du non-contact (0) entre les différents participants du réseau, sans identifier l'initiateur du contact.

Les différents indicateurs utilisés dans les analyses de la matrice permettent d'identifier les individus les plus importants du réseau. La centralité de degré relative mesure la position d'un individu en tenant compte de son nombre de connexions directes aux autres (Degenne et Forsé, 1994). La centralité d'intermédiarité mesure la faculté d'un individu de se situer sur le chemin de deux individus qui ne sont pas connectés et ainsi contrôler la communication entre eux. Par exemple, il est possible que l'individu A soit faiblement connecté aux autres, mais qu'il contrôle la communication entre $\mathrm{B}$ et $\mathrm{C}$, ce qui lui donne une influence non négligeable (Degenne et Forsé, 1994). Ce type de centralité réfère à la qualité des contacts qu'un individu a dans le réseau (Morselli, 2009). Les personnes ayant une forte centralité d'intermédiarité sont positionnées tels des courtiers entre les autres acteurs du réseau. Finalement, la centralité d'agglomération (clustering coefficient) mesure la capacité d'un individu de former des cliques, soit un regroupement de plus de deux personnes qui sont liées entre elles. En fait, cette mesure réfère à la densité locale du réseau qui indique à quel point les contacts directs d'un participant sont eux-mêmes connectés entre eux. Ainsi, cela identifie les formations de clique autour de participants.

L'analyse de réseau permet d'étudier les liens dans le réseau, mais ne permet pas de préciser clairement les éléments de contrôle. Ainsi, la présente étude utilise la méthode de Varese (2006) dans laquelle une 
analyse des conversations a mené à l'identification de tâches principales réalisées par les membres du réseau. Les conversations entre les 142 participants retenus pour l'analyse de réseau sont segmentées en fonction des différents thèmes de conversation, plusieurs thèmes pouvant se retrouver dans une même conversation téléphonique, par exemple le fait de maintenir l'ordre interne ou de gérer les tâches liées au travail de prostitution. Comme l'écoute électronique se déroule sur onze semaines, l'analyse des conversations est faite pour chaque semaine. Le nombre de segments de conversations ainsi que la diversité des sujets expliquent le choix du calcul par semaine et non par jour. Ces conversations segmentées par semaine sont aussi attribuées à la ou aux personnes en discutant durant la conversation téléphonique. Dès qu'un thème est discuté dans une conversation, il est donc noté pour la semaine concernée pour tous les individus ciblés ${ }^{2}$. En tout, six thèmes ont été retenus pour notre analyse.

\section{La structure globale et le positionnement des participants}

Le sociogramme (graphique 1) inclut les 142 individus retenus pour l'analyse. Les proxénètes sont illustrés par des cercles noirs. Les prostituées sont représentées par des carrés gris. Les proxénètes 1 et 2 ainsi que la prostituée 4 sont ceux qui ont le plus grand nombre de connexions avec des individus étant peu ou pas liés aux autres acteurs du réseau. Il faut noter que les deux proxénètes, les participants 1 et 2 , sont frères.

La centralisation de degré de l'ensemble du réseau est de $36 \%$ et la centralisation d'intermédiarité est de $45 \%$, ce qui indique qu'il y a d'autres participants dans le réseau qui sont des acteurs clés alors que l'enquête policière ne visait que les seuls proxénètes. Ces personnes sont bien visibles sur le graphique 1 : ce sont les proxénètes 1 et 2 ainsi que la prostituée 4 . Le coefficient d'agglomération (clustering coefficient) de l'ensemble du réseau est de $73 \%$, ce qui est élevé. Malgré le peu de cohésion sur le plan global, il y a tout de même une importante cohésion locale, autour de quelques individus. Un tel résultat suggère ainsi une tendance à former des cliques au sein du réseau.

2. La codification a été effectuée par l'auteure et une agente de recherche. Au départ, l'accord interjuge était de $88 \%$. Il y a alors eu ajustement de tous les segments de conversation par l'auteure et l'agent de recherche afin d'atteindre $100 \%$. 


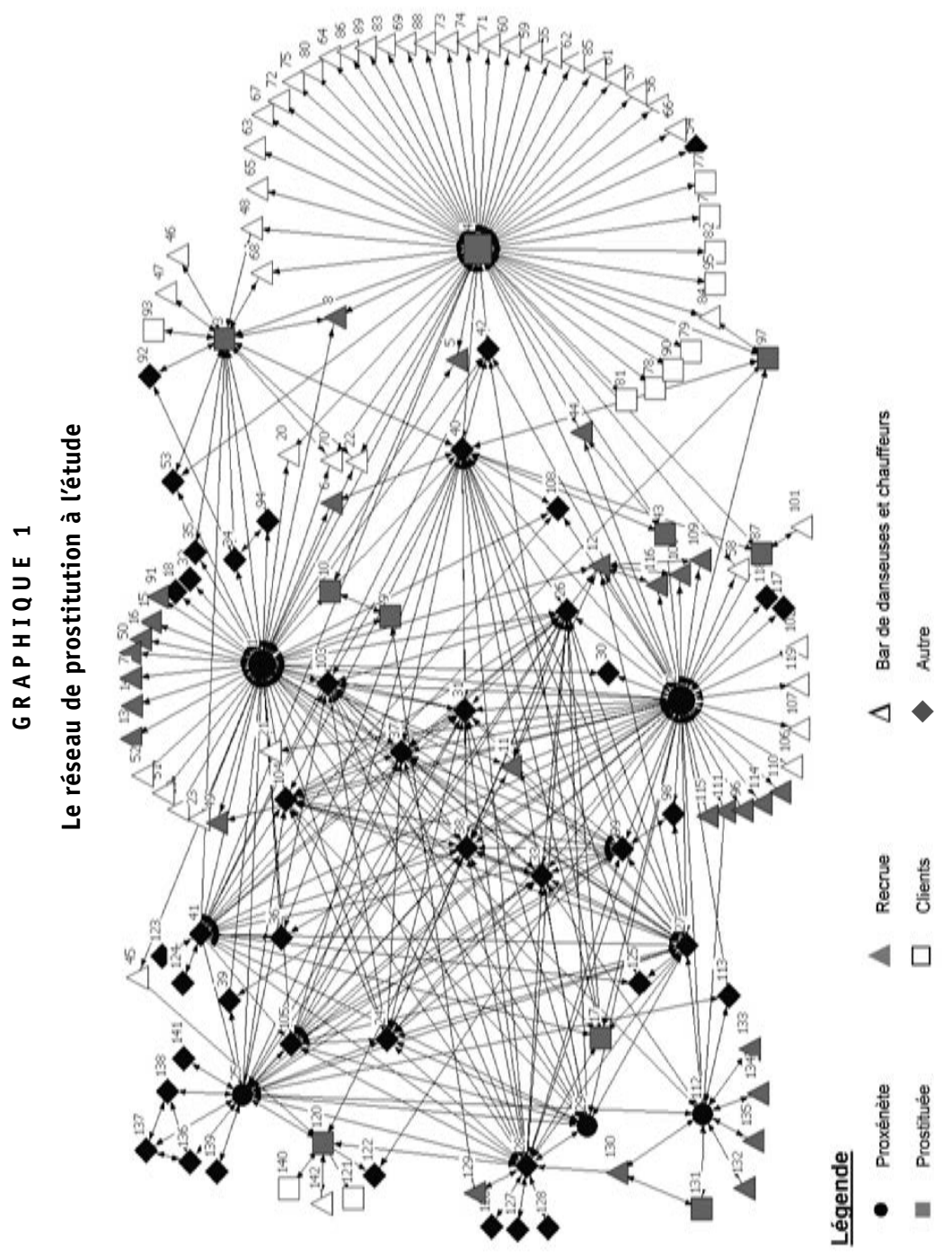


Ce sont les proxénètes 1 et 2 ainsi que la prostituée 4 qui ont le plus de contacts directs (centralité de degré respective de 39\%, 38,3\% et $36,2 \%$ ). Les proxénètes 1 et 2 ainsi que la prostituée 4 sont aussi les courtiers les plus importants (centralité d'intermédiarité respective de $36,5 \%, 32,1 \%$ et $45,9 \%$ ). Cela s'explique par les liens avec des acteurs indispensables à la réalisation du travail de prostitution: des chauffeurs, des clients, des agences de placement de danseuses et des bars de danseuses. La prostituée 4 est la prostituée qui entretient le plus ce genre de liens. Les proxénètes 1 et 2 , quant à eux, ont des contacts indispensables avec des recrues et des bars de danseuses, pour y faire travailler leurs prostituées et en trouver de nouvelles.

\section{Le proxénète 1 et ses prostituées}

Les participantes 3, 4, 9 et 10 sont les prostituées sous l'aile du proxénète 1 . La prostituée 4 travaille avec lui depuis environ trois ans. Il l'a recrutée lorsqu'elle était mineure. Elle a des contacts privilégiés avec le plus grand nombre de chauffeurs, de bars de danseuses et de clients. La prostituée 3 est dans la vie du proxénète depuis environ un an et elle a aussi été recrutée alors qu'elle était mineure. Pour ce qui est des prostituées 9 et 10 , elles commencent à travailler pour le proxénète 1 durant les semaines 7 et 9 de l'écoute électronique.

Que ce soit par rapport aux montants d'argent qu'elles amassent ou à la place privilégiée dans la vie du proxénète qu'elles veulent, il existe une compétition entre les prostituées 3 et 4 , les plus anciennes dans la vie du proxénète 1 . En effet, tout au long des conversations, la prostituée 4 émet des commentaires selon lesquels son proxénète lui aurait indiqué que, d'ici un an, elle pourrait arrêter de travailler et qu'ils commenceraient leur vie de couple exclusive. Elle croit occuper la première place dans la vie de son proxénète, qu'elle considère comme son chum. D'un autre côté, la prostituée 3 indique souvent à son amie, la participante 92 , qu'elle est la meilleure prostituée du proxénète 1 , qu'elle est celle qui rapporte le plus d'argent et qu'elle est la seule que le proxénète 1 aime vraiment. Elle ajoute fréquemment qu'il est son partner, ce qui a davantage une connotation d'affaires qu'amoureuse.

Pour ce qui est des montants d'argent que les prostituées du réseau amassent, la prostituée 3 est celle qui rapporte le plus d'argent, avec un minimum de 1000 \$ par quart de travail. La prostituée 4, quant à elle, fait entre $300 \$$ et $450 \$$ par soir. Toutefois, à un moment, les deux 
prostituées se mettent à travailler les mêmes quarts de travail au même endroit. La décision de faire travailler de concert les prostituées 3 et 4 est une idée du proxénète 1 après le départ, puis le retour, de la prostituée 3. En effet, à la fin de la cinquième semaine d'écoute électronique, celle-ci avait quitté le proxénète 1 pour un autre. À son retour, la semaine suivante, le proxénète a imposé ce travail en commun aux prostituées 3 et 4 . Ainsi, non seulement cela a instauré une compétition entre les filles pour rapporter plus d'argent, mais le proxénète peut aussi s'assurer des faits et gestes de la prostituée 3 en demandant à la prostituée 4 de la surveiller et de lui rapporter ses actions. De plus, cela réconforte la prostituée 4 quant à l'importance de sa position dans la vie du proxénète 1 .

Les rapports avec les prostituées 9 et 10 permettent de saisir comment s'effectue le début du travail des prostituées, prises sous l'aile du proxénète 1 . Au départ, elles font majoritairement du travail d'escorte, ce qui ne semble pas aussi payant que les bars de danseuses. En effet, au début, elles amassent au maximum 200 \$ par soir. Elles demandent en moyenne $60 \$$ par client. Le proxénète 1 , dès leur première semaine de travail, les motivent à faire plus d'argent. Ses techniques de persuasion semblent fonctionner puisque le montant amassé par soir augmente graduellement pour atteindre 400 \$ certains soirs. Vers la fin de la période d'écoute électronique, le proxénète 1 avance de plus en plus l'idée que les prostituées 9 et 10 commenceront à travailler dans les bars.

Habituellement, le proxénète 1 a des règles bien strictes. Les prostituées doivent lui téléphoner tous les soirs, qu'elles travaillent ou pas. Lors d'une soirée de travail, elles doivent l'appeler pour lui indiquer comment se déroule leur soirée, quelles sont les personnes avec qui elles ont eu des contacts et où elles en sont rendues côté financier. À la fin de leur quart de travail, elles doivent lui indiquer le montant total qui a été amassé et l'informer qu'elles quittent leur lieu de travail. Finalement, elles sont tenues de lui téléphoner lorsqu'elles sont rendues chez elle. Le proxénète 1 fait alors sa ronde; il va chez chacune d'elles et prend l'argent qu'elles ont gagné, leur laissant habituellement environ 80 \$ pour payer le chauffeur et le montant de base exigé par les bars pour laisser une fille danser, montant variant entre 50 \$ et $100 \$$. Toutefois, il faut savoir que le proxénète assume toutes les dépenses pour chacune de ses prostituées: l'appartement, la voiture, les meubles, les comptes, la nourriture, les vêtements, le bronzage, l'abonnement dans un centre sportif, les condoms, l'alcool et la drogue. Dès que la prostituée a besoin 
de quelque chose, elle n'a qu'à lui téléphoner et, dans la grande majorité des cas, il lui procurera ce qu'elle veut. Il ira souvent lui-même lui chercher ce dont elle a besoin.

D'autres règles bien strictes du proxénète 1 sont que les prostituées ne doivent pas parler à d'autres hommes noirs ${ }^{3}$, ni parler à ses anciennes prostituées, ni mentir, et qu'elles doivent dévoiler la somme exacte amassée chaque soir et lui remettre le montant demandé. En instaurant la règle de ne pas parler à d'autres hommes noirs, il réduit, selon lui, les risques de voir une de ses prostituées le quitter pour un autre proxénète. La règle de ne pas parler aux anciennes prostituées du proxénète 1 vise aussi à éviter que des calomnies à son sujet ne viennent aux oreilles de ses prostituées actuelles ou qu'elles soient recrutées par ses anciennes pour aller avec leur nouveau proxénète. Avec ces règlements, le proxénète 1 espère augmenter les chances que ses prostituées travaillent le plus longtemps possible avec lui. Le proxénète 1 est très sévère quant au respect des règles. Si une prostituée ne le fait pas, les punitions sont immédiates, quelles soient verbales, physiques ou en lui indiquant qu'il ne veut tout simplement plus travailler avec elle. Par exemple, une nouvelle recrue peut se faire remercier pour ses services par le proxénète quand il ne la trouve pas assez sérieuse, qu'elle ne rapporte pas assez d'argent et qu'elle ne se soumet pas assez à ses règles. Du côté des punitions physiques, lors d'une conversation interceptée, la prostituée 4 mentionne au proxénète 2 que le proxénète 1 lui a tiré les cheveux et l'a frappée parce qu'elle l'avait remis en question.

\section{Le proxénète 2 et ses prostituées}

Les prostituées du proxénète 2 sont les participantes 17, 43, 87 et 97 (graphique 1, dans le bas de l'image, au centre). La situation de la participante 17 est particulière en ce qu'elle pratique peu la prostitution, mais est plutôt utilisée comme prête-nom.

Pour ce qui est des prostituées 43,87 et 97 , le proxénète 2 a une façon bien particulière de les gérer. En effet, chaque semaine, une seule des trois prostituées reste à la maison pour prendre congé alors que les deux autres travaillent à l'extérieur de la ville ou même de la province. Durant leurs séjours à l'extérieur, elles vivent alors dans des motels. Ainsi, le proxénète peut consacrer tout son temps, chaque semaine, à la prostituée

3. Les proxénètes du réseau sont d'origine haïtienne. 
qui est auprès de lui. Il s'assure aussi que ses prostituées ne travaillent pas ensemble, ne voulant pas, au contraire du proxénète 1 , créer de compétition entre elles. Il tente d'assurer une belle entente avec chacune d'elles, en leur accordant à tour de rôle toute son attention.

La prostituée 43 est celle qui est dans la vie de son proxénète depuis le plus longtemps (cinq ans). Elle est proche de la prostituée 4. Elles se téléphonent presque tous les jours pour se donner des nouvelles. C'est surtout ainsi que des informations sur la prostituée 43 ont pu être amassées puisque son propre téléphone n'était pas sur écoute. La prostituée 4 connaît les autres prostituées du proxénète 2 et apprend aussi des informations sur celles-ci par l'entremise du proxénète 1 . Cela lui permet de tenir au courant son amie, ce qui n'est pas toujours à l'avantage du proxénète 2 puisque cela crée parfois une certaine jalousie.

La prostituée 97 est dans la vie du proxénète 2 depuis quatre ans alors que la prostituée 87 ne l'est que depuis deux ans. Le proxénète demande souvent à cette dernière si elle est certaine de vouloir être avec lui et lui demande fréquemment de lui donner sa confiance, une façon de s'assurer de son engagement envers lui, engagement qui semble parfois chancelant.

La prostituée 97, quant à elle, a déjà son appartement et se fait même payer une opération d'augmentation mammaire par le proxénète 2 . C'est une forme d'investissement puisqu'il indique à quelques reprises à sa mère, au proxénète 1 et à un autre proxénète avec qui il échange des informations sur le milieu, que ses prostituées ayant subi une telle chirurgie (les participantes 43 et 97) rapportent beaucoup plus d'argent qu'avant.

Les sommes d'argent amassées par les prostituées du proxénète 2 ont été plus difficiles à comptabiliser puisqu'il n'était pas aussi consciencieux et contrôlant que le proxénète 1 . En effet, il ne leur téléphonait pas tous les jours pour leur demander des comptes. Les rares fois où cela a été discuté, elles semblaient faire environ 200 \$ à 300 \$ par soir. À quelques reprises, le proxénète 2 indique que «des $200 \$, 300 \$$ lui donnent mal à la tête, qu'il veut entendre des 400 \$, 500 \$».

Le proxénète 2 a aussi certaines règles, mais elles réfèrent surtout à la consommation. Il ne veut pas que ses prostituées boivent et consomment beaucoup de drogues, surtout pas lorsqu'elles travaillent. Il leur demande souvent ce qu'elles ont consommé et, comme il les fournit en drogues, il leur indique combien de temps cela devrait leur prendre pour utiliser ce qu'il leur a donné. 


\section{Les autres proxénètes et leurs prostituées}

Le proxénète 25 est plus impliqué dans le trafic de stupéfiants que dans la prostitution. La prostituée 120 est dans sa vie depuis deux ans et travaille régulièrement sans avoir besoin d'être motivée par son proxénète. La seule règle stricte que le proxénète 25 lui demande de respecter est de lui téléphoner chaque jour, entre autres pour lui indiquer s'il a besoin qu'elle lui envoie de l'argent. En fait, c'est grâce aux revenus de sa prostituée, la participante 120 , que le proxénète 25 peut œuvrer dans le crime. Les deux autres proxénètes suspectés sont les participants 29 et 112. Le participant 29 étant en prison la plupart du temps d'écoute, il a peu de contacts dans le réseau alors que le participant 112 , ayant déjà été accusé de proxénétisme avant la mise sur écoute, n'a pas réellement de prostituées sous son aile.

\section{La mère}

Deux autres acteurs du réseau sont les parents des proxénètes 1 et 2 , ce sont les participants 40 (mère) et 41 (père). La mère des proxénètes est surtout importante. Elle entretient des liens particuliers avec certaines des prostituées de ses fils. Elle les coiffe régulièrement et profite de ces occasions pour les rassurer quant à l'amour que ses fils leur portent et ainsi les convaincre de rester dans la vie de ses fils et de leur remettre l'argent qu'elles font. Aux prostituées 4 et 43, elle fait miroiter une sécurité et un bel avenir, ce qui semble les aider à se raccrocher à leur vie avec les proxénètes 1 et 2 , lorsqu'elles en doutent. À quelques reprises, la mère dit même à l'un ou l'autre de ses fils: "Je lui ai fait un lavage de cerveau pour toi » afin de le persuader que ses prostituées ne le quitteront pas.

À ses fils, elle donne parfois des conseils sur la façon d'agir avec les prostituées qui semblent moins heureuses, qui semblent vouloir les quitter. Elle les motive à avoir des petites attentions pour chacune. Aussi, elle les met en garde contre certaines d'entre elles, par exemple les prostituées 3 et 87 , qui sont celles qui quittent leur proxénète respectif à un certain moment de l'écoute électronique. Elle indique à ses fils comment s'assurer qu'elles sont réellement motivées à rester avec eux et comment elles doivent prouver qu'elles sont dignes de confiance.

Ainsi, le rôle de la mère dans la vie de ses fils, les proxénètes 1 et 2 , est essentiel à l'évolution de ceux-ci comme proxénètes. Le proxénète 1 indique à plusieurs reprises que l'opinion de sa mère est importante, 
qu'il souhaite lui offrir un bel avenir. De plus, lorsque la prostituée 3 l'a quitté, il lui a dit: "[...] quand j'ai su que tu étais partie, j'avais tellement de la haine, tellement d'idées qui me passaient par la tête - je pensais à ma mère et je me suis dit que ça va la briser».

Ce n'est pas la première fois que l'on remarque l'importance de l'influence de la mère dans la vie des proxénètes. En effet, dans son autobiographie, Iceberg Slim (1967), un proxénète américain, indique comment sa mère a aidé à forger son identité et l'a motivé à vivre sa vie de proxénète.

\section{L'analyse des conversations}

L'analyse des conversations permet de saisir la nature des liens ainsi que les ressources échangées. Ces ressources reflètent les rôles et les tâches assumés principalement par treize acteurs formant le cœur du réseau, soit les trois proxénètes actifs, les neuf prostituées ainsi que la mère des deux proxénètes principaux, tels que présentés dans le graphique 2 . Ces acteurs sont liés à $84 \%$ du total des conversations pertinentes.

\section{GRAPHIQUE 2}

\section{Le cœur du réseau}

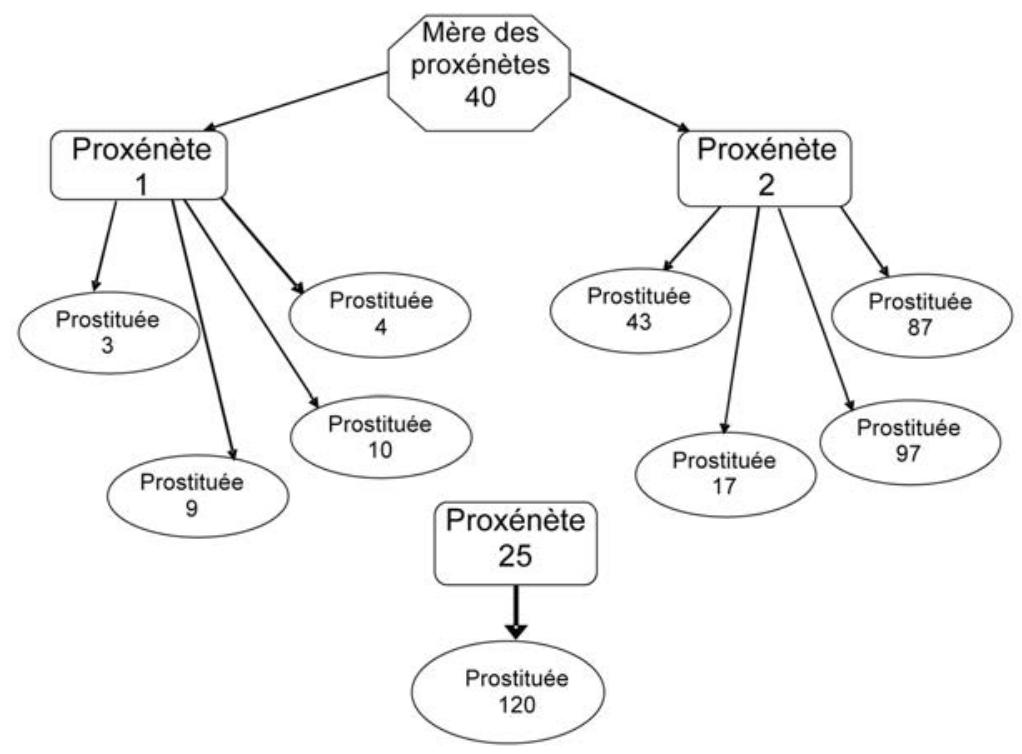


T A B LEA U 1

Dynamique des ressources utilisées par le cœur du réseau

\begin{tabular}{|c|c|c|c|c|c|c|c|c|c|c|c|c|c|c|}
\hline Thème & 1 & 2 & 25 & 40 & 4 & 3 & 9 & 10 & 43 & 87 & 97 & 17 & 120 & Total \\
\hline Protection & 10 & 8 & - & - & - & - & - & - & - & - & - & - & - & 18 \\
\hline $\begin{array}{l}\text { Maintien de l'ordre } \\
\text { interne }\end{array}$ & 622 & 176 & 24 & 142 & - & - & - & - & - & - & - & - & - & 964 \\
\hline $\begin{array}{l}\text { Gestion des finances } \\
\text { et du personnel }\end{array}$ & 499 & 208 & 66 & 63 & 12 & 1 & 2 & - & - & - & - & - & - & 851 \\
\hline Gestion des tâches & 13 & 9 & - & - & 526 & 97 & 8 & 6 & 34 & 19 & 5 & 3 & 25 & 745 \\
\hline $\begin{array}{l}\text { Acquisition de biens } \\
\text { matériels et } \\
\text { d'informations }\end{array}$ & 566 & 343 & 48 & 5 & 754 & 42 & 6 & 2 & 8 & 2 & 17 & 47 & 12 & 1852 \\
\hline Besoins à combler & - & - & - & - & 70 & 39 & 47 & 26 & 13 & 24 & 10 & 1 & 27 & 257 \\
\hline Total & 1710 & 744 & 138 & 210 & 1362 & 179 & 63 & 34 & 55 & 45 & 32 & 51 & 64 & 4687 \\
\hline
\end{tabular}


Six thèmes ont été définis durant l'analyse des conversations. Ceux-ci correspondent en partie à ceux étudiés par Varese (2006), avec certains ajustements pour s'adapter aux spécificités du milieu de la prostitution: la gestion des finances et du personnel, la gestion des tâches, le maintien de l'ordre interne, les besoins à combler des prostituées, la protection, ainsi que l'acquisition de biens matériels et d'informations. Le tableau 1 présente la fréquence des discussions des treize acteurs selon les différentes ressources. Les sous-totaux indiquent les segments de conversation, pour chaque ressource, pour chacune de ces personnes. Les résultats sont présentés en trois sections: les ressources utilisées exclusivement par les proxénètes, les ressources partagées entre les proxénètes et les prostituées, et, finalement, les ressources propres aux prostituées.

\section{Ressources propres aux proxénètes}

La protection réfère à toutes les actions ou les promesses d'action qui sont liées aux besoins de protection des prostituées. Il faut constater la rareté d'occurrence $(n=8$ et $n=10)$. Néanmoins, c'est la seule ressource qui est propre aux proxénètes, toutes les autres étant parfois réalisées par les prostituées ou la mère des proxénètes.

Le maintien de l'ordre interne est représenté par toutes les actions prises par les proxénètes pour conserver un contrôle sur leurs prostituées, pour assurer une certaine obéissance aux règles. Cela peut se faire par la manipulation, par les promesses d'un bel avenir, par la violence ou par les menaces. Dans ce réseau, les proxénètes ont chacun leur façon de fonctionner: le proxénète 2 opte plutôt pour la manipulation, notamment en culpabilisant ses prostituées de ne pas faire ce qu'il désire. Il dit souvent aux filles qu'il pense à se suicider ou qu'il est malheureux, qu'il veut juste être heureux et pouvoir compter sur elles. Ce genre de manipulation ressort souvent lorsqu'il sent qu'une prostituée s'éloigne de lui: «Bébé, abandonne-moi plus; je t'aime plus que tout.»

Le proxénète 1 , lui, fonctionne surtout avec les menaces et les coups. À quelques reprises, il a d'importants conflits avec les prostituées 3, 4 et 9 où il hurle dans le téléphone:

Je m'en viens te foutre une calotte. Reste là, j'arrive! Si je viens te voir, ça va être fucked up! Tu vois là, dans ces situations-là, moi, chus la mafia chus le lion qui s'est réveillé. Je vais te câlisser une de ces droites - tu pourras pas travailler pendant 3 jours! Je vais tellement te faire de bleus dans la face que tu te reconnaîtras même plus! 
Le troisième proxénète (25) ne semble pas avoir recours à une forme claire de manipulation ou de violence. Cependant, il s'assure régulièrement que ses demandes soient remplies, en les répétant et en téléphonant de nombreuses fois par jour à sa prostituée.

\section{Ressources partagées entre les proxénètes et les prostituées}

La première ressource partagée est la gestion des finances et $d u$ personnel, qui réfère à toutes les actions de gestion des prostituées et de l'argent amassé par celles-ci. Par exemple, les proxénètes vont souvent motiver les filles à travailler le plus fréquemment possible. Ils fixent aussi les montants d'argent qu'elles doivent rapporter durant une soirée ainsi que l'endroit où elles devraient travailler. Les proxénètes 1 et 2 sont très actifs sur ce plan, alors que le proxénète 25 l'est moins. En effet, sa prostituée, la participante 120, semble s'occuper seule de gérer les tâches liées au travail de prostitution, et ce, sans avoir besoin de la motivation de son proxénète.

Quelques-unes des prostituées s'acquittent aussi de certaines de ces tâches, mais dans une moins grande mesure. Par exemple, durant une certaine période, la prostituée 4 gère l'horaire de la prostituée 3. Pour ce qui est de la mère des proxénètes, elle gère parfois les finances de ses fils en leur demandant des comptes et les intimant de lui donner de l'argent ou d'en mettre de côté. Elle a aussi accès aux coffres-forts de ses fils.

La gestion des tâches se définit par toutes les actions visant à orchestrer les différents quarts de travail, par exemple les téléphones aux chauffeurs afin de réserver une place dans une voiture ou aux agences de placement de danseuses pour se réserver des heures de travail dans un bar de danseuses. La prostituée 4 est celle qui est la plus active de ce côté. Elle indique même au cours de certaines conversations qu'elle se sent proche de ses chauffeurs et de quelques personnes qui s'occupent de réserver ses heures de travail dans les bars de danseuses. Les proxénètes 1 et 2 sont peu concernés par ce genre de tâches.

L'acquisition de biens matériels et d'informations réfère entre autres à toutes les informations échangées qui permettent aux acteurs d'obtenir quelque chose. Par exemple, les informations échangées entre les proxénètes sur la façon de maintenir adéquatement l'ordre interne avec leurs prostituées ainsi que sur le fonctionnement des bars de danseuses sont une façon pour eux d'optimiser leur gestion. Les proxénètes 1 et 2 indi- 
quent à un moment que: «Les filles sont au courant de la game - si elles se chicanent, on va les battre. Aucune femme ne peut gâter la business.» Ils s'encouragent à garder un contrôle sur leurs prostituées afin de ne pas perdre le dessus. Ce thème réfère aussi au recrutement de nouvelles prostituées ou de nouveaux clients.

La prostituée 4 est celle qui acquiert le plus de ressources de ce type, notamment par tous les clients ou les clients potentiels avec qui elle est en contact ainsi que par les informations qu'elle échange avec d'autres prostituées. Elle est aussi celle qui obtient facilement de l'information puisqu'elle connaît presque toutes les prostituées du proxénète 2 . La prostituée 17 a un statut particulier pour ce qui est d'acquérir des ressources puisqu'elle loue souvent des appartements ou achète des voitures de luxe pour les proxénètes parce qu'elle a un bon crédit alors que les autres prostituées n'en ont aucun.

\section{Ressources propres aux prostituées}

Les besoins à combler sont toutes les demandes que les prostituées peuvent formuler à leur proxénète, comme être conduites quelque part, être rappelées, obtenir de l'argent ou simplement que leur proxénète vienne leur rendre visite. Les prostituées vont souvent accepter les demandes des proxénètes, en échange de faveurs, de services rendus par ceux-ci. Ainsi, elles réussissent à obtenir certains privilèges et plusieurs biens matériels qu'elles désirent. Plusieurs des conflits entre les proxénètes et leurs prostituées éclatent à cause des demandes constantes et répétitives des prostituées qui veulent être satisfaites. Les proxénètes doivent donc faire des compromis et leur donner ce qu'elles veulent, afin qu'elles, à leur tour, respectent les demandes des proxénètes. Cette ressource est clairement la source du levier des prostituées. Elles arrivent à leurs fins en faisant pression sur leur proxénète, en faisant des demandes pressantes. En observant le tableau 1, il est clair que les prostituées du proxénète 1 expriment plus de besoins à combler que les prostituées du proxénète 2 .

\section{Interprétation des résultats}

Deux constatations générales ressortent de l'analyse de ce réseau de prostitution. Elles reflètent les paradoxes qui sont en jeu dans ce dernier. 


\section{Entraide et compétition}

Il ressort une différence marquée dans les relations qu'entretiennent les proxénètes entre eux et celles que les prostituées vivent. Alors que l'on pourrait croire que les proxénètes sont très territoriaux et possessifs, dans ce réseau, une relation de collaboration et d'entraide est beaucoup plus présente. Le fait que les proxénètes 1 et 2 sont frères aurait pu expliquer une relation de collaboration. Toutefois, lorsque la prostituée 3 quitte le proxénète 1 pour un autre, les deux hommes en viennent à se parler pour convenir de laisser tranquilles leurs prostituées respectives, et ce, sans grande menace de part et d'autre. Le nouveau proxénète de la prostituée 3 a même indiqué que ce sont ses prostituées qui s'occupent du recrutement et que si elles ont joué dans les plates-bandes d'un autre proxénète en le sachant, elles seront punies comme il se doit. En effet, le proxénète 1 indique son intérêt et son respect envers l'autre proxénète: "Je disais justement à ma pute que si j'avais un ami comme toi, on pourrait collaborer et devenir millionnaires en un an. La paix est revenue, je t'invite à prendre une bière».

En fait, les personnes du réseau qui semblent le plus en compétition sont les prostituées. Plusieurs des conversations vont dans ce sens. Les seules prostituées qui semblent s'entraider sont les prostituées 4 et 43 qui échangent des informations sur les autres prostituées du réseau et se consolent lorsqu'elles se sentent abandonnées par leur proxénète. Les autres prostituées ne fonctionnent pas ainsi. C'est chacune pour soi afin de se rapprocher de leur homme. Il y a même des moments où le proxénète 1 tente lui-même de stimuler cette compétition. Par exemple, lorsqu'il demande à la prostituée 4 de travailler avec la prostituée 3 puisque cette dernière est revenue dans sa vie, la prostituée 4 n'est pas encline à accepter. Toutefois, elle le fait après que le proxénète 1 lui a dit: «Yo, je te dis où aller travailler pis tu fermes ta gueule - tu comprends pas- c'est quoi le problème!? Toé ta tête, c'est plein de marde dedans - tu comprends pas, OK?! T'es quel genre de femme - t'aimes pas la compétition?!

La compétition entre prostituées concerne aussi les prostituées de différents proxénètes. En effet, à un moment, la prostituée 43 indique, en parlant du travail de danseuses, que:

C'est important le prix que tu demandes à un client à cause de la compétition entre les filles. Si tu charges moins cher, tu peux manger une volée par les filles. Puis tu dois ramasser tes condoms souillés sinon 
tu as une amende de 100 \$ à payer au bar. Il y a même des filles qui jettent des condoms souillés dans la loge d'une autre fille pour l'écœurer et la faire payer. Certaines filles sont salopes et font vraiment tout.

\section{La prostituée, utilisée, mais indispensable}

Dans le débat à savoir si les prostituées sont exploitées ou pas, la présente étude permet de les considérer comme étant utilisées, mais indispensables. Par exemple, le proxénète 1 , surtout dans le cas de la prostituée 4, profite des ressources de ses prostituées et les utilisent, mais sans elles, il n'en aurait aucune lui permettant de réussir dans le milieu.

Un autre élément essentiel des relations entre proxénètes et prostituées est que cela prend un certain temps à former une prostituée. Il s'agit d'un investissement à long terme que le proxénète accepte de faire lorsqu'il sent que la prostituée est bien engagée envers lui. Les prostituées rapportant le plus et obéissant le mieux sont souvent celles qui sont depuis longtemps dans la vie du proxénète. Il est donc difficile pour un proxénète de retrouver une prostituée numéro 1 si jamais il la perd. C'est entre autres pour cela que le proxénète 1 fait autant de démarches pour ravoir la prostituée 3 .

Les prostituées sont peut-être utilisées pour leurs ressources, mais puisque ce sont elles qui s'occupent de tout ce qui a trait à la gestion des tâches liées au travail de prostitution, elles se constituent un capital propre. Éventuellement, si elles le veulent bien, elles pourraient utiliser ce capital uniquement à leur avantage et non plus en majeure partie pour le proxénète.

Ce sont celles qui sont dans la vie de leur proxénète depuis le plus longtemps qui ont le meilleur capital (les prostituées 3, 4 et 43). Elles s'acquittent même parfois de tâches faites habituellement par des proxénètes, par exemple lorsque la prostituée 4 gère le travail de la prostituée 3. De plus, à un moment, ces dernières, travaillant ensemble, se mettent même à recruter de concert une nouvelle prostituée pour leur proxénète. Il y a assurément une différence entre les plus jeunes prostituées nouvellement recrutées, par exemple les participantes 9 et 10, et celles qui sont bien établies dans la vie des proxénètes, les participantes 3, 4, 43, 87 et 97. 


\section{Conclusion}

Il faut reconnaître que la plupart des études antérieures ont présenté la relation entre un proxénète et ses prostituées comme en étant une d'exploitation, qu'elle soit conçue comme l'exploitation du corps de la femme ou encore comme son exploitation financière. Toutefois, si les proxénètes sont encore très présents dans le milieu de la prostitution, c'est que leur fonction et leur rôle leur permettent de rester actifs dans cet univers. Le proxénète répond donc à certains besoins des prostituées tels que l'amour, la protection, le besoin de se sentir en sécurité. Plus particulièrement, les relations d'affaires qui peuvent se créer entre un proxénète et une prostituée n'excluent pas l'existence d'un caractère amoureux dans la relation, ce qui peut cependant contribuer à la vulnérabilité de la prostituée et à son exploitation. Toutefois, tous ces besoins des prostituées n'expliquent pas nécessairement la fonction du proxénète ou les interactions entre les acteurs de ce milieu.

Même si plusieurs ressources sont partagées par les deux groupes, il y a tout de même des ressources qui sont respectivement propres aux proxénètes et aux prostituées. Ces ressources peuvent même être considérées comme étant les leviers de pouvoir dans leurs relations. Les proxénètes sont les seuls à pouvoir offrir de la protection et ils se basent sur cela pour motiver les prostituées à se joindre à eux alors que les prostituées retirent des gains de leurs relations avec leur proxénète, en leur faisant d'innombrables demandes matérielles et émotives que ceuxci acceptent de combler pour ne pas les perdre.

Une des limites les plus importantes de cette étude est que les conversations entre les acteurs du réseau ont uniquement pu être analysées durant onze semaines. Ce n'est qu'après huit mois d'enquête qu'a débuté l'écoute électronique. Selon les enquêteurs, un grand nombre de filles ont été en contact avec ces proxénètes, mais il n'est pas possible d'analyser ces relations, car elles n'ont pas eu lieu durant le temps d'écoute. De plus, après onze semaines, les enquêteurs ont eu assez de preuves pour accuser les proxénètes et cela a conduit à la cessation de l'écoute électronique. Toutefois, à ce moment, le réseau est en expansion. En effet, l'acquisition des biens matériels et des informations est alors à son plus haut niveau, ce qui indique que le réseau fonctionne au maximum. Les proxénètes ont plusieurs filles et certaines recrutent même pour eux.

Certains chercheurs indiquent que les raisons pour lesquelles une prostituée souhaite avoir un proxénète sont différentes selon que celle- 
ci est mineure ou majeure. Ainsi, les prostituées mineures citent plus souvent des besoins sentimentaux que les prostituées adultes qui indiquent plus souvent des besoins de gestion ou de protection. Ces différences peuvent alors expliquer les types de relations entre proxénètes et prostituées (Bracey, 1979).

Dans ce réseau, les prostituées ont toutes été recrutées alors qu'elles étaient mineures, mais la majorité de celles-ci étaient adultes au moment de la mise sous écoute. Les données recueillies ne permettent pas de réellement saisir les motivations exactes de chacune des prostituées. Toutefois, la prostituée 43 , une adulte, mentionne qu'elle: «[...] reste avec lui pour la motivation. Sans mon chum, je ne travaillerais pas autant et ne ferais donc pas beaucoup d'argent. Quand tu n'as pas le choix, tu fais plein d'argent.

Le cadre d'analyse de cette étude reconnaît l'importance des interactions d'échange entre un proxénète et ses prostituées. Il offre ainsi une perspective qui permet de déterminer si la prostituée est dominée ou pas par son proxénète et quel est le rôle de celui-ci dans ce processus d'échange. Dans ce type de partenariat, l'acteur central du réseau, le proxénète, force les prostituées en partenariat avec lui à évoluer dans un contexte de compétition entre elles. Cet environnement peut sembler favoriser le proxénète qui maintient un certain contrôle sur ses prostituées. Toutefois, en évaluant la qualité de ces relations, au-delà de la structure d'ensemble et à travers le temps, il apparaît que le partenaire qui semble le plus faible conserve un certain pouvoir, notamment en demandant au proxénète de combler ses besoins. Le message implicite est, qu'en fait, les prostituées peuvent contrôler la façon dont les proxénètes les contrôlent.

\section{Références}

Adler, P. A. (1985/1983). Wheeling and dealing: ethnography of an upper-level drug dealing and smuggling community. $2^{\text {nd }}$ edition. New York: University Press, New York.

Augustin, L. M. (2005). The cultural study of Commercial Sex. Sexualities, 8 (5), 618-631.

Barry, K. (1995). Prostitution of sexuality. New York: University Press.

Bell, L. (1987). Good girls bad girls: Sex-trade workers and feminists face to face. Toronto: The Women's Press.

Block, A. (1979). The snowman cometh: Coke in progressive New York. Criminology, 17, 75-99. 
Bracey, D. H. (1979). “Baby-Pros”: Preliminary profiles of juvenile prostitutes. New York: John Jay Press.

Caplan, G. M. (1984). The Facts of Life about Teenage Prostitution. Crime and Delinquency, 30 (1), 69-74.

Dalla, R. L., Xia, Y., \& Kennedy, H. (2003). You just give them what they want and pray they don't kill you: Street-level sex workers' reports of victimization, personal resources, and coping strategies. Violence Against Women, 9 (11), 1367-1394.

Degenne, A., \& Forsé, M. (1994). Les réseaux sociaux. Paris: Armand Colin.

Desroches, F. J. (2005). The crime that pays: Drug trafficking and organized crime in Canada. Toronto: Canadian Scholars Press.

Eck, J. E., \& Gersh, J. S. (2000). Drug trafficking as a cottage industry. In M. Natarajan et M. Hough (eds.), Crime Prevention Studies (241-271). Monsey: Criminal Justice Press.

Enablers, Inc. (1978). Juvenile Prostitution in Minnesota (Report of a research project). Minneapolis: University of Minnesota.

Haller, M. H. (1990). Illegal enterprise: A theorical and historical interpretation. Criminology, $28(2), 207-235$.

Hodgson, J. F. (1997). Games pimps play: Pimps, players and wives-in-law. A qualitative analysis of street prostitution. Toronto: Canadian Scholars' Press.

Jacobs, B. A. (1999). Dealing crack: The social world of street corner selling. Boston: Northeastern University Press.

Lacasse, S. (2003). Le travail des danseuses nues: au-delà du stigmate, une relation de service marchand (Thèse de doctorat). Université de Montréal.

May, T., Harocopos, A., \& Hough, M. (2000). For love or money: Pimps and the management of sex work. London: Research, Development and Statistics Directorate.

McLeod, E. (2003). Eroticizing prostitute use. In R. Matthews \& M. O’Neill (eds.), Prostitution (145-188). Burlington: Aldershot.

Milner, C., \& Milner, R. (1972). Black players. Boston: Little, Brown and Company.

Morselli, C. (2009). Inside criminal networks. New York: Springer.

Norton-Hawk, M. (2004). A comparison of pimp-and non-pimp-controlled women. Violence Against Women, 10 (2), 189-194.

Pearson, G., \& Hobbs, D. (2001). Middle market drug distribution. London: Home Office.

Pheterson, G. (1996). The prostitution prism. Amesterdam: Amsterdam University Press.

Raphael, J., \& Shapiro, D. L. (2004). Violence in indoor and outdoor prostitution venues. Violence Against Women,10 (2), 126-139.

Shelby, T. (2002). Parasites, pimps, and capitalists: A naturalistic conception of exploitation. Social Theory and Practice, 28 (3), 381-418.

Slim, I. (1967). Pimp: The story of my life. London: Payback Press. 
Svedgi, A. O., St-Lauwrence, J. S., Tikhonova, L., Safarova, E., Parker, K. A., Shakarishvili, A., \& Ryan, C. A. (2003). The social organization of commercial sex in Moscow, Russia. Sexually Transmitted Diseases, 30 (1), 39-45.

Varese, F. (2006). The structure of criminal network examined: The RussianItalian Mafia in Rome. Oxford Legal Studies Paper, 21.

Weisberg, K. D. (1985). Children of the night. A study of adolescent prostitution. Toronto: Lexington Books.

Williamson, C., \& Cluse-Tolar, T. (2002). Pimp-controlled prostitution: Still an integral part of street life. Violence Against Women, 8 (9), 1074-109. 
ABSTRACT - The study focuses on the roles, interactions, and resource exchange process at the core of a prostitution network. The analysis illustrates how pimps and prostitutes contribute in different ways to a common network. Such an exchange system becomes the basis for understanding the structure of order and control within the network. This approach allows us to examine how prostitutes are not mere subordinates to pimps and, in many ways, occupy key positions and roles of privilege within the overall network. Thus, whereas the traditional focus on pimp and prostitute networks has maintained that pimps generally have complete control over prostitutes, our study demonstrates how prostitutes may control how others control them. The data for this study is based on electronic surveillance transcripts that were intercepted during a law-enforcement investigation that targeted the network under analysis. After demonstrating how the network was structured and how both pimps and prostitutes maintained key positions therein, a conversational analysis of all relevant telephone calls that were intercepted over a two-year period is pursued. Results demonstrate that the roles that prostitutes occupy in the network and the resources that they contribute make them vital participants in the overall structure of order. Analysis of such positions, roles, and resource sharing reveal the competitive environment that prostitutes create against one another and their indispensable value to the network beyond the evident servicing of clients. Such findings and insights are relevant for sex and human trafficking settings as well as general contexts of criminal networking.

KEYWORDS - Pimp, prostitution, criminal networks, social network analysis, conversation analysis.

RESUMEN - El presente estudio trata sobre los roles, las interacciones y el intercambio de recursos en el seno de una red de prostitución desmantelada por un servicio policial de una gran ciudad canadiense. Más que centrar el debate en la explotación de las prostitutas por los proxenetas, el análisis de dicha red ha sido realizado desde el punto de vista del uso compartido de recursos, que es frecuentemente utilizado por otro tipo de redes criminales. Dicha perspectiva tiene en cuenta la manera en la que la prostituta es o no dominada por su proxeneta, y cómo esto ha de determinar el rol del susodicho en el proceso de intercambio. El mensaje implícito es que las prostitutas pueden tener un cierto control sobre las formas en que los otros las controlan. Un análisis de las posiciones de los individuos en la red es aplicado con el fin de distinguir los participantes centrales y estratégicos. Un análisis de las conversaciones es considerado el método más indicado para el examen de las redes criminales, lo que posibilita discernir el rol de cada prostituta y de cada proxeneta, así como también sus formas de interacción. El estudio concluye que los proxenetas tienden más a ayudarse entre sí que las prostitutas, quienes se encuentran en una competición constante. Finalmente, mismo si los proxenetas utilizan el capital de las prostitutas para forjarse el suyo, éstas resultan indispensables, ya que un proxeneta sin prostituta no puede existir, si bien la inversa es posible. Dicha realidad procura a las prostitutas una forma significativa de poder en las relaciones con sus proxenetas.

PALABRAS CLAVE - Proxeneta, prostitución, red criminal, análisis de redes, análisis de conversaciones. 